\title{
Direct measurement of the image displacement instability in a linear induction accelerator
}

\author{
T. J. Burris-Mog, C. A. Ekdahl, and D. C. Moir \\ Los Alamos National Laboratory, Los Alamos New Mexico 87545, USA
}

(Received 14 December 2016; published 19 June 2017)

\begin{abstract}
The image displacement instability (IDI) has been measured on the $20 \mathrm{MeV}$ Axis I of the dual axis radiographic hydrodynamic test facility and compared to theory. A $0.23 \mathrm{kA}$ electron beam was accelerated across 64 gaps in a low solenoid focusing field, and the position of the beam centroid was measured to 34.3 meters downstream from the cathode. One beam dynamics code was used to model the IDI from first principles, while another code characterized the effects of the resistive wall instability and the beam breakup (BBU) instability. Although the BBU instability was not found to influence the IDI, it appears that the IDI influences the BBU. Because the BBU theory does not fully account for the dependence on beam position for coupling to cavity transverse magnetic modes, the effect of the IDI is missing from the BBU theory. This becomes of particular concern to users of linear induction accelerators operating in or near low magnetic guide fields tunes.
\end{abstract}

DOI: 10.1103/PhysRevAccelBeams.20.060403

\section{INTRODUCTION}

Understanding instabilities that can cause unwanted behavior for a beam of charged particles in an accelerator is important for both new accelerator designs and characterizing stable parameters of existing machines. One of these instabilities is the image displacement instability (IDI) which causes a displacement of the beam centroid from the accelerator axis, effectively steering the beam [1-3]. Two conditions must be met for the instability to occur. First, the accelerated beam is high current and/or in a low magnetic guide field. Second, there must be a discontinuity within the accelerator walls such as accelerating gaps. This latter point makes the instability especially concerning for users of linear induction accelerators (LIAs) [4-6] and for present designers of new LIAs such as the Los Alamos National Laboratory, the Institute of Fluid Physics (China Academy of Engineering Physics) [7], and the Budker Institute of Nuclear Physics (Russian Academy of Sciences) [8].

Although previous experiments have explored the IDI [9], it appears that the measurements presented here are the first to decisively validate the IDI theory. Typically, users of an LIA suppress the beam break-up (BBU) instability by increasing the strength of the magnetic guide field, and in doing so also suppress the IDI. However, there are times when operating with a low magnetic guide field is desired, making the IDI a concern. An additional result of these

Published by the American Physical Society under the terms of the Creative Commons Attribution 4.0 International license. Further distribution of this work must maintain attribution to the author(s) and the published article's title, journal citation, and DOI. measurements is that it appears the IDI amplifies the BBU instability, as the present BBU theory predicts a significantly slower BBU growth rate compared to these measurements.

\section{IDI THEORY}

The IDI arises from an imbalance of electric and magnetic forces resulting from electrons traveling near a discontinuity in a conducting surface. These two competing forces arise as a beam of electrons traveling through a conducting beam pipe generates both image charge and image current within the pipe walls. The image charge in turn generates an electric field that acts to displace the beam centroid from the axis, while the image current generates a magnetic field that acts to restore the beam centroid to the axis (Fig. 1).

In sections of the conducting beam pipe, these two forces cancel to within $\gamma^{-2}$, where $\gamma$ is the Lorentz factor of the electron beam. However, when the beam passes through a discontinuity, such as an LIA accelerating gap, the image charge builds up on the gap corners while the image current must travel around the perimeter of the gap. Although the image charge is not present in the gap, its buildup at the gap corners causes the electric field to remain nearly unchanged, but the more distant image current diminishes the magnetic field responsible for holding the beam centroid to the axis. These two opposing forces are azimuthally symmetric only for beam centroids traveling on axis, so a perturbation of the beam in the transverse direction will lead to a steering effect.

By combining the Biot-Savart law with the Lorentz force, the transverse force acting on the beam due to the magnetic field can be written as (in Gaussian units), 


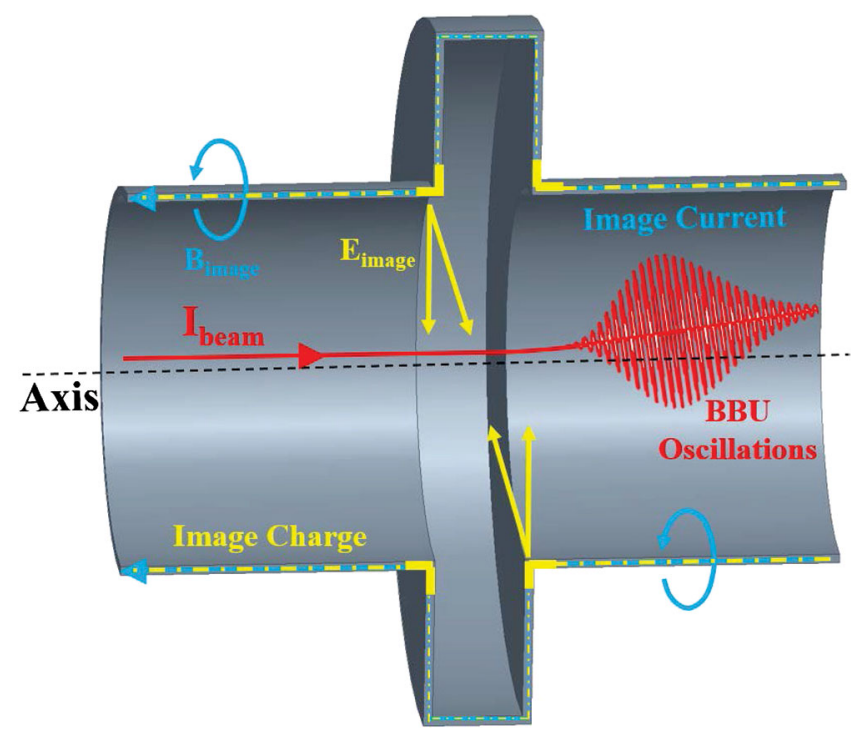

FIG. 1. A beam of charged particles traveling down a conducting beam pipe generates image charge and image current. The image charge generates an electric field with a force acting to pull the beam away from the axis. The image current generates a magnetic force acting to push the beam toward the axis. When the beam is off axis, these forces are asymmetric. The IDI is responsible for steering the beam centroid. The BBU instability is responsible for imprinting and amplifying oscillations in the beam.

$$
F_{x}=\frac{2 q v I}{c^{2} r}=\frac{2 q v I x_{i}}{c^{2} b^{2}}
$$

where $I$ is the beam current, $q$ is the electron charge, $v$ is the axial velocity of the electrons, and $b$ is the radius of the beam pipe. The transverse centroid displacement of $x_{i}$ results in the magnetic image current being located at $r=b^{2} / x_{i}$. It is the removal of this force that allows the electric field to displace the centroid further off axis.

Since the change in transverse momentum is small, it is assumed that the position of the beam centroid across the gap remains constant. The transverse change in momentum across a gap of width $w$ is then,

$$
\Delta p_{x}=\frac{2 q w I x_{i}}{c^{2} b^{2}}
$$

A centroid initially offset by $x_{i}$ at the beginning of a gap will have a final displacement $x_{f}$ at the end of the gap which follows the matrix equation,

$$
\left[\begin{array}{l}
x_{f} \\
x_{f}^{\prime}
\end{array}\right]=\left[\begin{array}{cc}
1 & 0 \\
F^{-1} & 1
\end{array}\right]\left[\begin{array}{l}
x_{i} \\
x_{i}^{\prime}
\end{array}\right]
$$

where $x^{\prime}=p_{x} / p_{z}$ is the angle of the beam centroid. Combining $p_{x}$ and $p_{z}=\gamma m(\beta c)$ yields,

$$
\frac{1}{F}=\frac{2 q w I}{\gamma \beta m c^{3} b^{2}}=\frac{1}{17.1[\mathrm{kA}]}\left(\frac{2 w I}{\gamma \beta b^{2}}\right)
$$

which can be thought of as an effective (negative) focal strength, since it steers the centroid off axis. The beam current $I$ is in units of kilo-amperes, all lengths are in units of centimeters, and $m c^{3} / q=17.1 \mathrm{kA}$ because, in this case, the beam is composed of electrons.

With this effective (negative) focal strength, the minimum magnetic field required to suppress the IDI by a solenoid field can be found by relating the values of the centroid's offset and angle ( $x$ and $\left.x^{\prime}\right)$. The solenoid's matrix equation can be written as,

$$
\left[\begin{array}{l}
x_{f} \\
x_{f}^{\prime}
\end{array}\right]=\left[\begin{array}{cc}
\cos (\theta) & 2 \rho \sin (\theta) \\
-\sin (\theta) / 2 \rho & \cos (\theta)
\end{array}\right]\left[\begin{array}{c}
x_{i} \\
x_{i}^{\prime}
\end{array}\right]
$$

where, $\rho=m c^{3} \gamma \beta / e B=1.7 \gamma \beta / B$ for $B$ in units of kiloGauss, $\theta=L /(2 \rho)$, and $L$ in centimeters is the distance between gaps.

The criteria for stable transverse motion then requires,

$$
\left|\cos (\theta)+\frac{\rho}{F} \sin (\theta)\right|<1
$$

The expression inside the absolute value bars is found by multiplying the four by four matrices from Eq. (3) and Eq. (5) and then taking the trace of the result. With a little manipulation and use of the trigonometric identity $[1-\cos (\theta)] / \sin (\theta)=\tan (\theta / 2), \rho / F<\tan (\theta / 2)$, which becomes $\rho / F<(\theta / 2)$ for small deflection angles as is the case in IDI. Inserting the values for $\rho$ and $1 / F$ and solving for $B$, we find the minimum solenoid field required to suppress the IDI is then,

$$
B>\frac{1}{b} \sqrt{\frac{1.36 I \gamma \beta w}{L}} .
$$

We would like to note that Eq. (7) can also be found by writing the equation of motion for the electron beam as a Mathieu equation and solving for the stable solutions [10].

\section{ISOLATING THE IDI}

During normal operations at Axis-I of the DARHT facility, the magnetic guide fields are set well above the IDI suppression threshold given in Eq. (7). We refer to such tunes as nominal tunes and an example can be seen in Fig. 9 of Ref. [11]. Magnetic fields below the IDI suppression threshold were applied on Axis I [12] of the DARHT facility. Axis I is a single-pulse linear induction accelerator with 64 ferrite filled induction cells. Each cell has a gap width $w=1.91 \mathrm{~cm}$ and a cell to cell spacing of $43.2 \mathrm{~cm}$, while the radius of the beam pipe is $7.6 \mathrm{~cm}$. For this experiment a beam current of $0.23 \mathrm{kA}$ with a $60 \mathrm{~ns}$ flat-top 


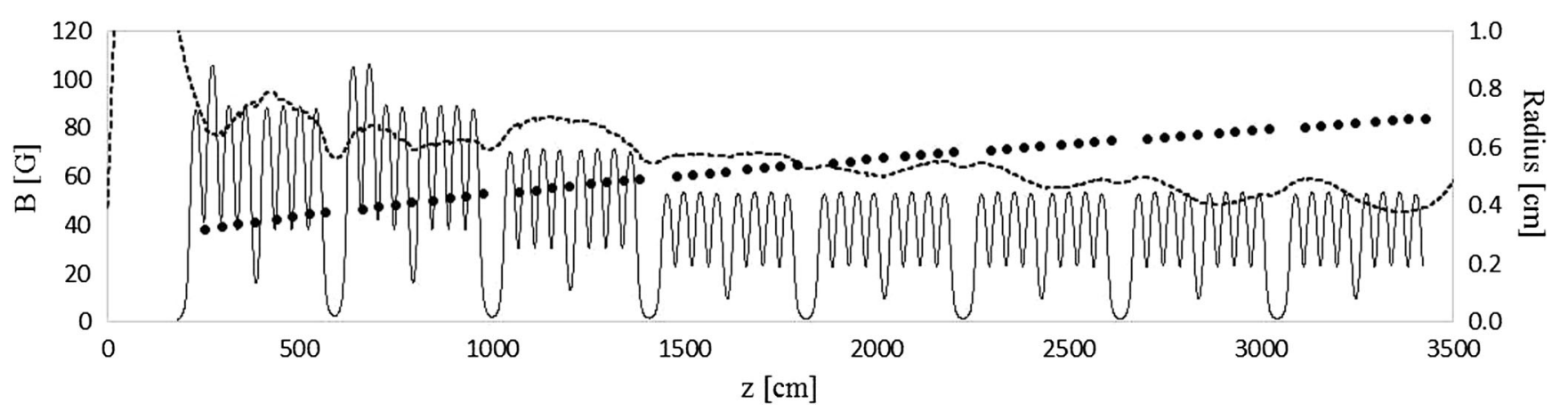

FIG. 2. Left Axis-The axial magnetic field of the Axis I solenoids (solid line) and the magnetic field needed to suppress the IDI as calculated by Eq. (7) (dots). Right Axis-The radius of the electron beam (dashed line).

was injected at $3.3 \mathrm{MeV}$, and each induction cell further imparted $250 \mathrm{keV}$ onto the electrons.

Each induction cell houses a direct current solenoid which generates the magnetic fields used to guide the electrons to the end of the accelerator. Figure 2 shows the magnetic guide field along the axis of the accelerator. The induction cells are grouped in sets of eight, and the areas of low magnetic field are drift spaces. Missing from this figure is the field from the anode magnet which is located $54 \mathrm{~cm}$ downstream from the cathode and focuses the beam with 570 Gauss. The dots in Fig. 2 show the minimum magnetic field required to suppress the IDI, as calculated from Eq. (7). As the electrons gain energy, the field required to suppress the instability grows, i.e. $B \propto \sqrt{\gamma \beta}$.

Under these conditions, theory predicts that the IDI is large enough to overcome the focusing effects of the solenoids. The measured radial offset of the centroid for the $0.23 \mathrm{kA}$ beam traveling through this magnetic guide field is presented in Fig. 3 (dots). As we can see, the steering effect is increasing as the electrons gain energy and the guide fields remain low.

Using two beam dynamics codes, we compare measurement and theory and explore the relationship between the IDI and the BBU instability [13-18]. The first code, XTR [19], was designed to model the transport of electron beams on Axis I. For the IDI measurements, XTR was modified to include the theory presented above, but it does

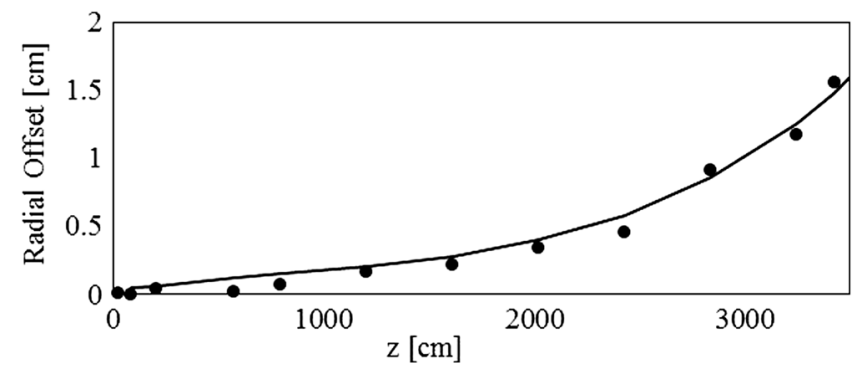

FIG. 3. Measured radial offset on Axis I (dots), and modeled IDI relying on the theory presented in Ref. [2] for an initial offset of $0.045 \mathrm{~cm}$ (solid line). The accuracy of the measurements is within $200 \mu \mathrm{m}$. not include the BBU instability. It also predicts the beam envelope shown in Fig. 2 (right axis) and accounts for the magnetic guide fields, gap voltages (i.e. electrostatic lensing effects) and beam scraping. Figure 3 shows that the results of the model (solid line) agree well with the measured data (dots). The second code [20,21], the Linear Accelerator Model for DARHT (LAMDA), has been benchmarked against theory, experimental data and other beam dynamics codes and was used to help understand what effect the BBU and resistive wall instabilities have on the IDI measurements.

The resistive wall instability [22-24] is similar to the IDI in that it arises from the imbalance of forces from the image charge and image current. Where the IDI occurs in discontinuities, the resistive wall instability occurs in continuous parts of the accelerator and leads to an attractive force toward the beam pipe wall. Because the magnetic field follows a characteristic diffusion time, this instability can lead to the additional effect of a displacement between the head and the tail of the beam. An initial offset of $1 \mathrm{~mm}$ was used in the LAMDA model, and Fig. 4(A) shows that the final centroid displacement is less than two millimeters and the head-to-tail displacement is even smaller. The resistive wall instability is therefore negligible compared to the IDI.

The BBU instability, however, is more dangerous as it can have a direct impact on the beam centroid measurements. The BBU instability occurs because the beam excites the transverse modes inside the accelerating cavities of an LIA, which in turn imprint an rf oscillation on the beam. Equation (1) in Ref. [25] shows that for an initial displacement of $\xi_{0}$ and an initial Lorentz factor of $\gamma_{0}$, the amplitudes of the BBU oscillations grow exponentially as,

$$
\xi=\xi_{0} \sqrt{\frac{\gamma_{0}}{\gamma}} e^{\Gamma}
$$

where $\Gamma=3.33 \times 10^{-3} I N_{g} Z_{\perp}\left\langle B^{-1}\right\rangle$ with current $I$ in kA, transverse impedance $Z_{\perp}$ in $\Omega / \mathrm{cm},\left\langle B^{-1}\right\rangle$ in $\mathrm{kG}^{-1}$ with the brackets indicating an average and $N_{g}=64$ gaps. The growth rates for nominal accelerator operation and for that of the IDI measurements are presented in Fig. 4(B). 

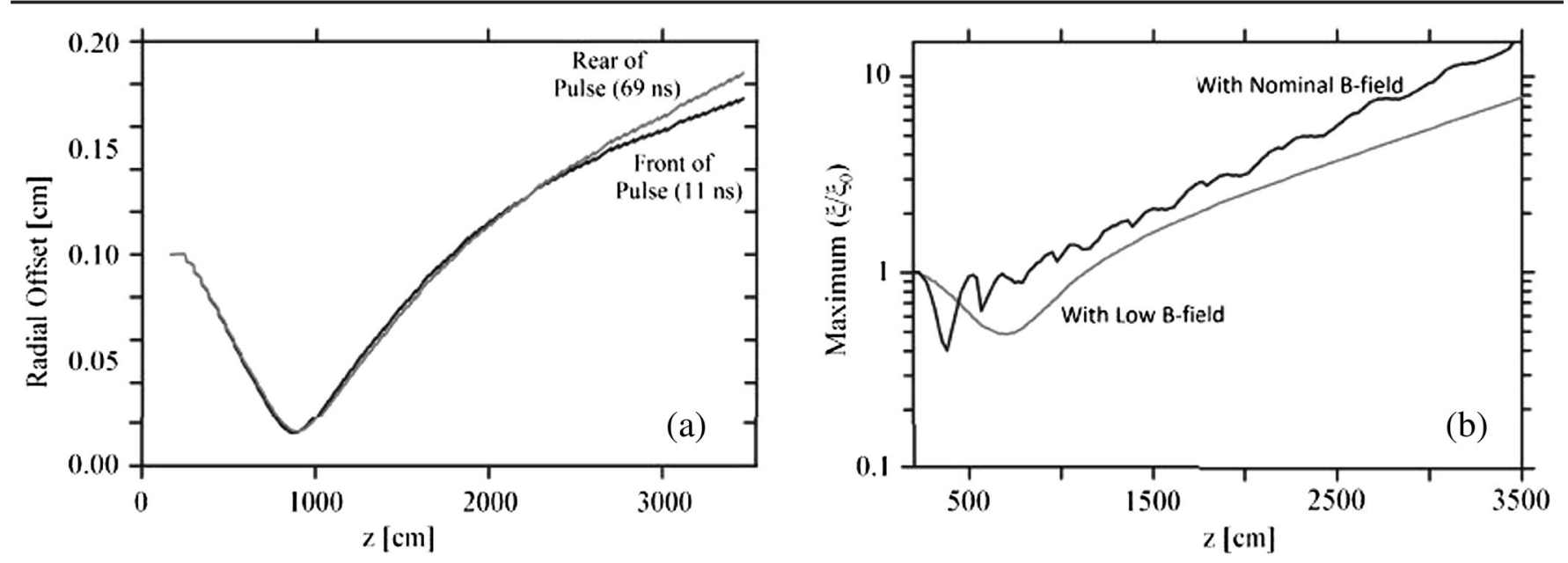

FIG. 4. LAMDA Code Calculations: (A)—The resistive wall instability and centroid displacement due to image currents and image charges in continuous parts of the accelerator lead to a final centroid displacement of $2 \mathrm{~mm}$ and a relative head to tail displacement of $0.1 \mathrm{~mm}$, both for an initial $1 \mathrm{~mm}$ centroid offset. (B)—The growth rate of the BBU instability for the IDI tune is lower than that of the nominal tune of Axis I.

The dip in Figs. 4(A) and 4(B) indicate that the BBU and resistive wall instabilities are suppressed by the magnetic fields early in the accelerator. In comparison with the low field tune, the nominal tune in Fig. 4(B) shows a much more rapid suppression of the BBU instability early in the accelerator along with oscillations on the curve. This more rapid suppression and oscillations are due to the much stronger focusing by the magnetic fields of the nominal tune. However, as the electron beam travels downstream, grows and, if large enough, leads to beam scraping and charge loss at the beam pipe walls. A charge loss to one side would then shift the centroid position and influence the IDI measurements. To be sure that the BBU growth was not shifting the beam centroid, we turn to the beam current measurements presented in Fig. 5A. The loss of charge begins around 34.3 meters. Since this is the point at which we stop the IDI measurements, we conclude that the BBU did not affect the measurements and the centroid displacement is a result of the IDI.

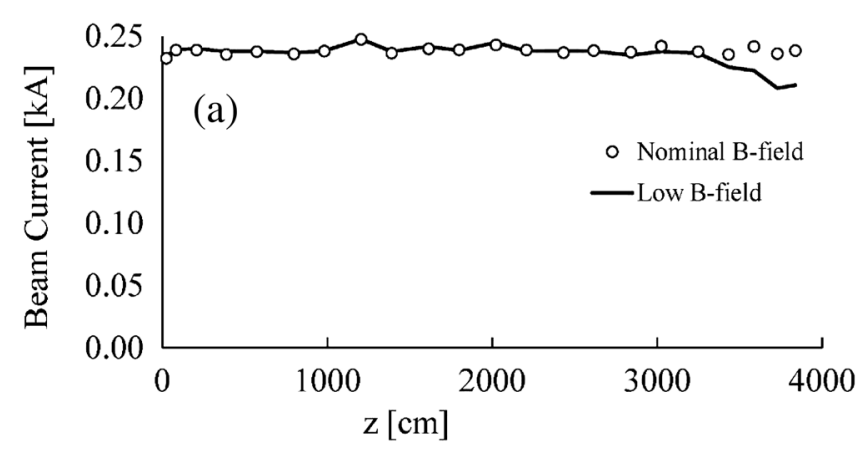

\section{AMPLIFICATION OF BBU}

The loss of charge to the beam pipe wall at the end of the accelerator is a surprise. The raw data for the low magnetic guide field case presented in Fig. 5(B) shows that the erosion of the beam current occurs at the rear of the pulse, which indicates the presence of the BBU instability at the end of the accelerator. As shown earlier in Fig. 4, the BBU growth rate for the low magnetic guide field was calculated to be lower than the growth rate for the nominal guide field, yet the measured BBU instability is present only in the low field case. As seen in Fig. 2, the radius of the beam is about $5 \mathrm{~mm}$ at the end of the measurements, and from Fig. 3, the final centroid displacement is $1.6 \mathrm{~cm}$. The XTR model, which includes the IDI but not the BBU instability, finds that the edge of the beam is at most $2.1 \mathrm{~cm}$ from the axis and well away from the $7.6 \mathrm{~cm}$ radius of the beam pipe wall. If we calculate the BBU growth rate using an initial displacement of $\xi_{0}=1 \mathrm{~mm}$ in the LAMDA model [Fig. 4(B)], which includes the BBU instability but not the

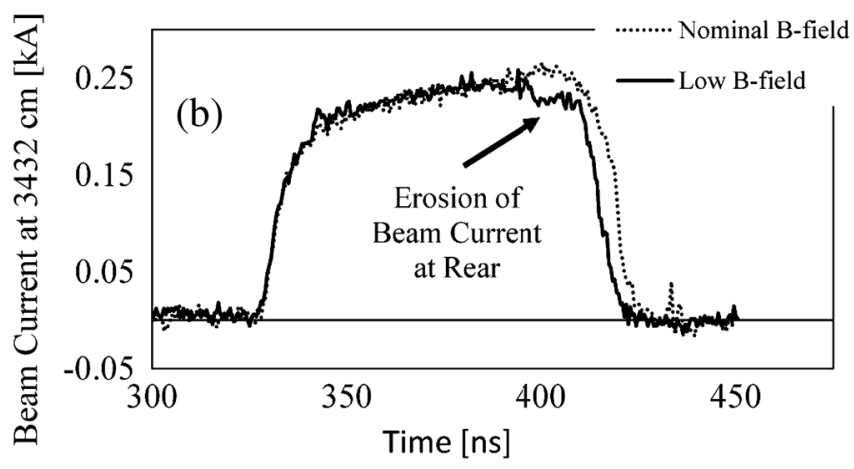

FIG. 5. (A) Beam current measurements along the length of the accelerator for nominal (dots) and low (solid line) magnetic guide fields. (B) Raw data from the nominal (dots) and low (solid line) magnetic guide field cases shows erosion of the beam current from the rear of the low field pulse which is indicative of the BBU instability. 
IDI, then the beam is at most $2.8 \mathrm{~cm}$ from the axis. The computer models predict that the BBU instability should not be severe enough to cause loss of beam current to the beam pipe wall, even at the end of the accelerator. The measurements in Figs. 5(A) and 5(B), however, show that beam current is lost at the end of the accelerator, and the computer models underestimated the severity of the BBU instability.

We consider two reasons why the present BBU theory did not predict the measured BBU growth. First, consider Eq. (8). The only displacement in the model is accounted for by the prefactor $\xi_{0}$. The displacement during the experiment, however, is growing due to the IDI. Second, the transverse resonant magnetic field responsible for the $\mathrm{BBU}$ instability is proportional to $\mathrm{J}_{1}$, the first order Bessel function, which is zero on axis and increases with radius. However, the BBU theory considers only a volume average [13], and LAMDA does not account for the dependence of the coupling field on beam position. Because of this, it seems reasonable to consider that the IDI is amplifying the BBU through the increasing displacement and that our present computer models may need revision to accurately predict BBU growth for off-axis beams.

\section{CONCLUSION}

The beam centroid measurements made on Axis I appear to be the first of their kind and compare well with analytical theory and computational analysis for the IDI. Analysis shows that the resistive wall and BBU instabilities were not significant enough to affect these measurements, and that the IDI was the driving force behind the beam steering. These measurements support the theory behind Eq. (7), which determines the minimum magnetic field needed to stabilize the IDI.

Furthermore, it appears that the BBU instability is amplified by the IDI. This reinforces the practice of using LIA tunes with magnetic fields purposely set high enough to stabilize the IDI. Experiments are being developed for Axis I to validate the stability threshold, and to further explore how the BBU is affected by the IDI.

\section{ACKNOWLEDGMENTS}

We would like to acknowledge the members of the DARHT technical and operations teams for their support during these experiments. This work was supported by the US National Nuclear Security Agency and the US Department of Energy under Contract No. DE-AC5206NA25396. (LA-UR-16-29375).

[1] C. H. Woods, The Image Instability in High Current Linear Accelerators, Rev. Sci. Instrum. 41, 959 (1970).

[2] V. K. Neil, Lawrence Livermore Laboratory Internal Report No. UCID-17838, 1978.
[3] V. K. Neil, Lawrence Livermore Laboratory Internal Report No. UCID-17976, 1978.

[4] E. Merle, R. Boivinet, M. Mouillet, J. C. Picon, O. Pierret, P. Anthouard, J. Bardy, C. Bonnafond, A. Devin, and P. Eyl, Status of the airix accelerator, in Proceedings of the 18th Particle Accelerator Conference, New York, 1999 (IEEE, New York, 1999), vol. 5, pp. 3260-3262.

[5] M. J. Burns, G. J. Caporaso, B. E. Carlsten, Y. Chen, K. P. Chow, E. G. Cook, H. A. Davis, C. A. Ekdahl, W. M. Fawley, C. M. Fortgang et al., Status of the dual axis radiographic hydrodynamics test (darht) facility, AIP Conf. Proc. 650, 139 (2002).

[6] J. Deng, B. Ding, H. Wang, G. Dai, N. Chen, L. Zhang, K. Zhang, Z. Dai, and J. Shi, Physical design of the dragon-i linear induction accelerator, High Power Laser Part. Beams 15, 502 (2003).

[7] J. J. Deng, J. S. Shi, and W. P. Xie, $R$ and $D$ status of highcurrent accelerators at IFP, J. Korean Phys. Soc. 59, 3619 (2011).

[8] D. A. Starostenko, A. V. Akimov, P. A. Bak, M. A. Batazova, A. M. Batrakov, Y. M. Boimelshtein, D. Y. Bolkhovityanov, A. A. Eliseev, A. A. Korepanov, G. I. Kuznetsov et al., Status of the LIA-2. Double-pulse mode, Phys. Part. Nucl. Lett. 13, 962 (2016).

[9] R. Adler, B. Godfrey, M. Campbell, D. Sullivan, and T. Genoni, The image-displacement effect in intense electron beams, Part. Accel. 13, 25 (1983).

[10] G. Caporaso and Y.-J. Chen, Induction Accelerators, Particle Acceleration and Detection, edited by $\mathrm{K}$. Takayama and R. Briggs (Springer-Verlag, New York, 2011), ISBN 978-3-642-13916-1.

[11] C. Ekdahl, Los Alamos National Laboratory Internal Report No. LA-UR-14-27454, 2014.

[12] J. E. Coleman, D. C. Moir, C. A. Ekdahl, J. B. Johnson, B. T. McCuistian, G. W. Sullivan, and M. T. Crawford, Increasing the intensity of an induction accelerator and reduction of the beam breakup instability, Phys. Rev. ST Accel. Beams 17, 030101 (2014).

[13] V. K. Neil, L. S. Hall, and R. K. Cooper, Further Theoretical Studies Of The Beam Breakup Instability, Part. Accel. 9, 213 (1979).

[14] R. B. Miller, J. W. Poukey, B. G. Epstein, S. L. Shope, T. C. Genoni, M. Franz, B. B. Godfrey, R. J. Adler, and A. Mondelli, Beam transport issues in high current linear accelerators, IEEE Trans. Nucl. Sci. 28, 3343 (1981).

[15] G. Caporaso, A. Cole, and K. Struve, Beam breakup (bbu) instability experiments on the experimental test accelerator (eta) and predictions for the advanced test accelerator (ata), IEEE Trans. Nucl. Sci. 30, 2507 (1983).

[16] G. J. Caporaso, The Control of Beam Dynamics in High Energy Induction Linacs, in Proceedings, 1986 Linear Accelerator Conference: Stanford, CA (1986), pp. MO2-1.

[17] R. J. Briggs, D. L. Birx, G. J. Caporaso, V. K. Neil, and T. C. Genoni, Theoretical and Experimental Investigation of the Interaction Impedances and $Q$ Values of the Accelerating Cells in the Advanced Test Accelerator, Part. Accel. 18, 41 (1986).

[18] Y. Y. Lau, Classification of Beam Breakup Instabilities in Linear Accelerators, Phys. Rev. Lett. 63, 1141 (1989). 
[19] P. Allison, D. C. Moir, G. Sullivan, and T. P. Hughes, Observation of self-steering effects on the its 6-mev linac, in Proceedings of the Particle Accelerator Conference, Vancouver, BC, Canada, 1997 (IEEE, New York, 1997), Vol. 1, p. 1144.

[20] T. Hughes, C. Mostrom, T. Genoni, and C. Thoma, Voss Scientific technical report No. VSL-0707, 2007.

[21] C. Ekdahl, J.E. Coleman, and B. T. McCuistian, Beam Breakup in an Advanced Linear Induction Accelerator, IEEE Trans. Plasma Sci. 44, 1094 (2016).
[22] S. Bodner, V. K. Neil, and L. Smith, Decay of image currents and some effects on beam stability, Part. Accel. 1, 327 (1970).

[23] G. Caporaso, W. Barletta, and V. Neil, Transverse resistive wall instability of a relativistic electron beam, Part. Accel. 11, 71 (1980).

[24] C. Ekdahl, Transient decay of beam-produced fields in thin-walled tubes, J. Appl. Phys. 89 (2001).

[25] C. Ekdahl, Electron-beam dynamics for an advanced flashradiography accelerator, IEEE Trans. Plasma Sci. 43, 4123 (2015). 